\title{
Pebble Sets in Convex Polygons
}

\author{
Kevin Iga • Randall Maddox
}

Received: 9 December 2005 / Revised: 29 July 2007 /

Published online: 8 September 2007

(C) Springer Science+Business Media, LLC 2007

\begin{abstract}
Lukács and András posed the problem of showing the existence of a set of $n-2$ points in the interior of a convex $n$-gon so that the interior of every triangle determined by three vertices of the polygon contains a unique point of $S$. Such sets have been called pebble sets by De Loera, Peterson, and Su. We seek to characterize all such sets for any given convex polygon in the plane.

We first consider a certain class of pebble sets, called peripheral because they consist of points that lie close to the boundary of the polygon. We characterize all peripheral pebble sets, and show that for regular polygons, these are the only ones. Though we demonstrate examples of polygons where there are other pebble sets, we nevertheless provide a characterization of the kinds of points that can be involved in non-peripheral pebble sets. We furthermore describe algorithms to find such points.
\end{abstract}

\section{Introduction}

Lukács and András posed the following in [1]: Prove that there exists a set $S$ of $n-2$ points in the interior of a convex $n$-gon such that for any three vertices of the $n$ gon, the interior of the triangle determined by the three vertices contains exactly one element of $S$. Many solutions to this problem were given, one of which was published in [2]. In [3], De Loera, Peterson, and Su employ analogous sets in $d$-dimensional polytopes to prove a generalization of Sperner's Lemma. Following the terminology in [3], we will call a solution to the question posed in [1] a pebble set.

In this paper, we consider the problem of characterizing all pebble sets in a given convex $n$-gon. We begin by characterizing a certain class of pebble sets, which we call peripheral, since the points are near the boundary of the polygon. For some kinds of

K. Iga (凶) · R. Maddox

Natural Science Division, Pepperdine University, 24255 Pacific Coast Hwy, Malibu,

CA 90263-4321, USA

e-mail: kiga@pepperdine.edu 
polygons, such as regular polygons, these are in fact the only pebble sets, but for other kinds of polygons, there are others. If we take a certain natural notion of equivalence of pebble sets, then this provides a lower bound to the number of pebble sets as a function of $n$.

In some cases, there may be pebble sets that are not peripheral, and we study necessary and sufficient conditions for such to exist. We also give a construction for analyzing polygons in which such pebble sets exist, breaking any such set down into pebble sets in smaller polygons. This allows us to characterize pebble sets for many polygons.

We begin in Sect. 2 with a more precise statement of the problem and some preliminary remarks about pebble sets. This section also contains terminology and notation that will be used throughout.

Section 3 introduces a construction that provides peripheral pebble sets, and proves that all peripheral pebble sets are of this type. Section 4 deals with nonperipheral pebble sets, giving a necessary and sufficient condition for such to exist. Here we also prove that there are no such pebble sets when the polygon is regular. In Sect. 5, we provide an efficient algorithm for determining if this condition is satisfied. Finally, in Sect. 6, we consider questions for further research.

\section{Preliminaries}

In this paper all polygons are assumed to be convex. In general, we will label the vertices and edges of an $n$-gon in the counterclockwise direction with the sequence $\left\langle v_{0}, e_{0}, \ldots, v_{n-1}, e_{n-1}\right\rangle$. We consider the subscripts for the vertices and edges as integers modulo $n$, so that for instance $v_{n}=v_{0}$ and $e_{n}=e_{0}$.

We will say that three vertices are consecutive if they are of the form $v_{k}, v_{k+1}$, $v_{k+2}$, and that two vertices are adjacent if they are of the form $v_{k}$ and $v_{k+1}$. A vertex is incident with an edge if it is an endpoint of the edge. Two edges are incident if they are of the form $e_{k}$ and $e_{k+1}$. By a chord of a polygon, we mean the segment joining two non-adjacent vertices of the polygon.

Definition 1 A pebble set $S$ in a convex $n$-gon $P$ in the plane is a set of $n-2$ points in the interior of $P$ so that every triangle determined by vertices of $P$ contains exactly one point of $S$ in its interior.

First we note the following.

Proposition 1 If $S$ is a pebble set in an n-gon $P$, then no point in $S$ lies on a chord of $P$.

Proof Let $p$ be a point on a chord $f$ in $P$, and consider a triangulation of $P$ that includes $f$. This triangulation contains $n-2$ triangles with pairwise disjoint interiors. Thus no pebble set can contain $p$.

By a chamber of a polygon, we mean a maximal connected subset of the polygon that does not intersect any edges or chords of the polygon. We will say that two 
subsets of the polygon are equivalent if the sets of chambers containing points in the subsets are identical. Clearly if $S$ is a pebble set that contains a point $p$ in a chamber $C$, and if $q$ is any point in $C$, then $[S-\{p\}] \cup\{q\}$ is also a pebble set. Therefore the property of being a pebble set depends only on the equivalence class.

With a slight abuse of notation, we will refer to an entire equivalence class of pebble sets as a pebble set, and note that we could construct pebble sets by merely specifying an appropriate set of chambers.

For convenience, when we refer to a triangle as a subset of the plane, we actually mean the interior of the triangle, since points in a pebble set by definition lie in the interiors of the triangles.

If $u, v$ and $w$ are distinct vertices in a polygon, we will refer to the triangle they form as $\Delta u v w$. We will refer to the triangle formed by an edge or chord $e$ and vertex $v$ as $\Delta e v$, and the triangle formed by two incident edges or chords $e$ and $f$ as $\Delta e f$.

If $v_{k-1}, v_{k}, v_{k+1}$ are consecutive vertices, then we call $\Delta v_{k-1} v_{k} v_{k+1}$ a border triangle, and we will refer to this triangle as the border triangle at $v_{k}$. We call the union of the border triangles the periphery of the polygon. The complement of the periphery in the polygon will be called the core of the polygon.

Since there are $n$ border triangles, then the fact that a pebble set contains $n-2$ points necessitates that at least two points in a pebble set will lie in the intersection of two overlapping border triangles. We will say that such a point lies close to an edge of the polygon. If a pebble set contains precisely two points that are close to an edge of the polygon, then the remaining points in the pebble set must be distributed among the remaining border triangles in a one-to-one correspondence, revealing that all points in the pebble set lie in the periphery. More generally, the pigeonhole property leads us to the following observation.

Proposition 2 If a pebble set in an $n$-gon contains $k \geq 2$ points that are close to an edge of the polygon, then there are precisely $k-2$ points in the pebble set that lie in the core.

The following will be helpful in showing that a set of points is a pebble set.

Lemma 3 Suppose $S$ is a set of $n-2$ points in an $n$-gon $P$, none of which lies on a chord. Let $\mathcal{T}$ be the set of triangles in $P$ of the form $\Delta e v$, where $e$ is an edge and $v$ a vertex of $P$. If every triangle in $\mathcal{T}$ contains at most one point of $S$, then $S$ is a pebble set.

Proof First we show that every triangle in $\mathcal{T}$ contains precisely one point of $S$. Let $T \in \mathcal{T}$. Then there exist an edge $e$ and a vertex $v$ such that $T=\Delta e v$. Triangulate $P$ with all chords from $v$. Every triangle in this triangulation is in $\mathcal{T}$, and thus contains at most one point of $S$. Since there are $n-2$ triangles in this triangulation, and since no point of $S$ lies on a chord of $P$, we have that $T$ contains exactly one point of $S$.

Now we show that $S$ is a pebble set. Let $T$ be any triangle determined by three vertices of $P$. If $T \in \mathcal{T}$, then $T$ contains precisely one point of $S$. So suppose $T \notin \mathcal{T}$. Then $T=\Delta v_{i} v_{j} v_{k}$, where no two of $\left\{v_{i}, v_{j}, v_{k}\right\}$ are adjacent. Assume that $0 \leq i<$ $j<k \leq n-1$. Working counterclockwise, triangulate $P$ with chords from $v_{i}$ to all vertices $v_{i+2}$ to $v_{j}$, chords from $v_{j}$ to all vertices $v_{j+2}$ to $v_{k}$, and chords from $v_{k}$ to 
Fig. 1 Shaded region is the fan of edge $e$

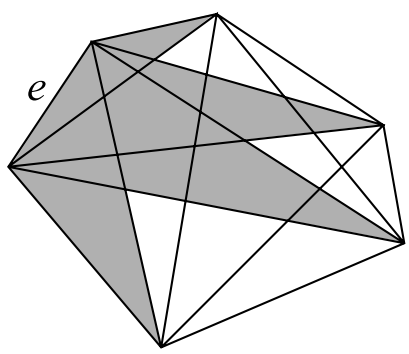

Fig. 2 A periphery pebble set for an 11-gon where $A=\{1,2,4,6\}$

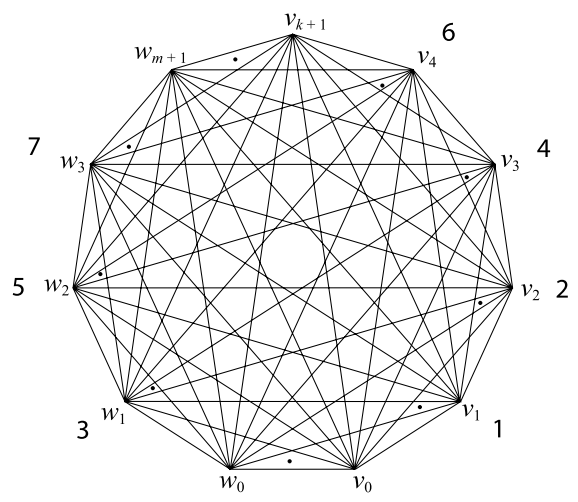

all vertices $v_{k+2}$ to $v_{i}$. Every triangle except $\Delta v_{i} v_{j} v_{k}$ in this triangulation contains precisely one edge of $P$, and therefore contains precisely one point of $S$. Since there are $n-3$ triangles in the triangulation other than $\Delta v_{i} v_{j} v_{k}$, and since no point of $S$ lies on a chord of $P$, there must be precisely one point of $S$ in $\Delta v_{i} v_{j} v_{k}$.

Definition 2 For a given edge $e$ of a polygon, the union of all triangles of the form $\Delta e v$ across all vertices $v$ of $P$ is called the fan of $e$. (See Fig. 1.)

Lemma 3 implies that it is only necessary to check that the triangles in the fans of the edges of a polygon contain at most one point of a set $S$ to determine if $S$ is a pebble set.

\section{Characterizing Peripheral Pebble Sets}

We say that a pebble set is peripheral provided all points in the pebble set lie in the periphery. We present a construction whereby all peripheral pebble sets of an $n$-gon can be obtained.

Construction 1 Given any subset of $W=\{1,2, \ldots, n-4\}$, we may determine a unique pebble set that contains a point close to edge $v_{0} v_{n-1}$ in the following way.

First let $A$ be a subset of $W$, and suppose $A$ has $k$ elements. (See Fig. 2.) Let $B=W-A$, so that $B$ has $m=n-4-k$ elements. We begin by relabeling some of 
the vertices and edges of $P$ for convenience. For $0 \leq i \leq m+1$, we let $w_{i}=v_{n-1-i}$. Label edges $f_{i}=w_{i} w_{i+1}$ for $0 \leq i \leq m$. For $0 \leq i \leq k$, let $T_{i}$ denote the border triangle at $v_{i}$, and for $0 \leq i \leq m$, let $U_{i}$ denote the border triangle at $w_{i}$.

Note that each $T_{i}$ is divided into $n-2$ chambers by the chords emanating from $v_{i}$. We label these chambers $C_{i, j}(0 \leq j \leq n-3)$ in the following way. For $0 \leq i \leq k$, we let $C_{i, 0}$ be the chamber close to $e_{i-1}$, labeling consecutively up to $C_{i, n-3}$, the chamber close to $e_{i}$. Similarly for the $U_{i}(0 \leq i \leq n-3)$, we let $D_{i, 0}$ be the chamber of $U_{i}$ close to $f_{i-1}$, labeling up to $D_{i, n-3}$, the chamber close to $f_{i}$.

To construct a pebble set, begin by letting $p_{0}$ be a point in the chamber close to $v_{0} w_{0}$, and $p_{n-3}$ a point in the chamber close to $v_{k+1} w_{m+1}$. Writing the elements of $A$ as $a_{1}<a_{2}<\cdots<a_{k}$, let $p_{i}$ be a point in each $C_{i, a_{i}}$. Similarly, writing the elements of $B$ as $b_{1}<b_{2}<\cdots<b_{m}$, let $p_{i+k}$ be a point in each $D_{i, b_{i}}$. Let $S=\left\{p_{0}, \ldots, p_{n-3}\right\}$.

Theorem 4 Let $P$ be an $n$-gon $(n \geq 4)$ and $e$ an edge of $P$. Then the construction described above determines a one-to-one correspondence between the peripheral pebble sets containing a point close to the edge $v_{0} w_{0}$ and the power set of $\{1,2, \ldots, n-4\}(n \geq 4)$.

Proof We use induction to show that $S$ as constructed above is a pebble set. If $n=4$, then $A=B=\emptyset$. Thus $S$ consists of a point close to edge $v_{0} w_{0}$ and a point close to $v_{1} w_{1}$, which is clearly a pebble set. So suppose $n \geq 5$ and that this construction produces a pebble set for a polygon on fewer than $n$ vertices. Note that we may assume that $1 \in A$. For otherwise, an argument similar to the one to follow can be applied to $B$.

First we let $n^{\prime}=n-1$ and create an $n^{\prime}$-gon, which we will denote $P^{\prime}$, by deleting $v_{0}$ and all chords of $P$ emanating from it, then including the segment $w_{0} v_{1}$ as an edge of $P^{\prime}$. (See Fig. 3 for an example.) We show that $S^{\prime}=S-\left\{p_{0}\right\}$ is precisely the same construction in $P^{\prime}$ as the above construction is for $P$. To demonstrate this, we relabel some of the vertices, border triangles, and chambers of $P^{\prime}$, as well as the points in $S^{\prime}$ to reveal a subset $A^{\prime}$ of $W^{\prime}=\left\{1,2, \ldots, n^{\prime}-4\right\}$ that has $k^{\prime}=k-1$ elements, a set $B^{\prime}=W^{\prime}-A^{\prime}$, and a selection of points in the chambers of the border triangles of $P^{\prime}$ analogous to that for $P$.

For $0 \leq i \leq k^{\prime}+1$, let $v_{i}^{\prime}=v_{i+1}$ and $e_{i}^{\prime}=e_{i+1}$. For $1 \leq i \leq n^{\prime}-3$, let $p_{i}^{\prime}=p_{i+1}$. First note that $S^{\prime}$ contains $p_{0}^{\prime}=p_{1}$, which is close to edge $v_{0}^{\prime} w_{0}$, and it contains

Fig. 3 Deletion of $v_{0}$ from $P$ in the proof of Theorem 4

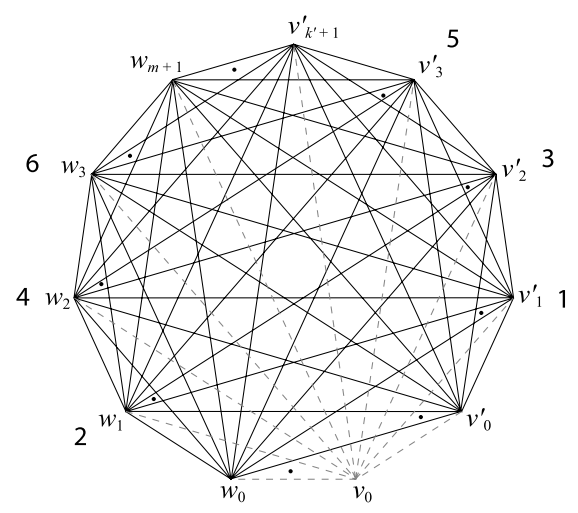


$p_{n^{\prime}-3}^{\prime}=p_{n-3}$, which is close to $v_{k^{\prime}+1}^{\prime} w_{m+1}$. Label the border triangles at the $v_{i}^{\prime}$ as $T_{i}^{\prime}$, and note that $T_{i^{\prime}}=T_{i+1}$. Label the chambers of $T_{i}^{\prime}$ as $C_{i, j}^{\prime}$ as before, where $C_{i, 0}^{\prime}$ is the chamber in $T_{i}^{\prime}$ close to $e_{i-1}^{\prime}$, labeling consecutively up to $C_{i, n^{\prime}-3}$, the chamber of $T_{i}^{\prime}$ close to $e_{i}^{\prime}$. With this labeling, we have that $C_{i, j}^{\prime}=C_{i+1, j+1}$ for $i \leq j \leq n^{\prime}-3$. Let $A^{\prime}=\{a-1: a \in A, a \neq 1\}$ and label the elements of $A^{\prime}$ as $a_{i}^{\prime}=a_{i+1}-1$ for $1 \leq$ $i \leq k^{\prime}$. Note that $A^{\prime}$ is a subset of $W^{\prime}=\left\{1,2, \ldots, n^{\prime}-4\right\}$ that contains $k^{\prime}$ elements. Furthermore, since $a_{i} \geq i$, we have that $a_{i}^{\prime} \geq i$. Also, $C_{i, a_{i}^{\prime}}^{\prime}=C_{i+1, a_{i}^{\prime}+1}=C_{i+1, a_{i+1}}$, so that $C_{i, a_{i}^{\prime}}^{\prime}$ contains $p_{i}^{\prime}=p_{i+1}$.

In a somewhat similar fashion, we relabel the chambers in $P^{\prime}$ of the border triangles at the $w_{i}(0 \leq i \leq m)$, where $D_{i, 0}$ is the chamber of $U_{i}$ close to $f_{i-1}$, up to $D_{i, n^{\prime}-3}$, the chamber of $U_{i}$ close to $f_{i}$. With this relabeling, $D_{i, j}^{\prime}=D_{i, j+1}$ for $i \leq j \leq n^{\prime}-3$. Let $B^{\prime}=\{b-1: b \in B\}$, and label the elements of $B^{\prime}$ as $b_{i}^{\prime}=b_{i}-1$ for $1 \leq i \leq m$. Note that $B^{\prime}=W^{\prime}-A^{\prime}$. Since $1 \in A$, we have that $b_{i} \geq i$ for $1 \leq i \leq m$. Also, $D_{i, b_{i}^{\prime}}^{\prime}=D_{i, b_{i}^{\prime}+1}=D_{i, b_{i}}$, so that $D_{i, b_{i}^{\prime}}^{\prime}$ contains $p_{i+k^{\prime}}^{\prime}=p_{i+k}$.

With this, we have that the removal of $v_{0}$ from $P$ leaves an $n^{\prime}$-gon whose set of points $S^{\prime}$ is of the same construction as that for $P$. We now show that $S$ is a pebble set in $P$, for $n \geq 5$ by applying Lemma 3 .

Let $e$ be any edge of $P$ and $v$ any vertex. We show $\Delta e v$ contains precisely one point of $S$. If $e \notin\left\{v_{0} w_{0}, e_{0}\right\}$, and $v \neq v_{0}$, then $\Delta e v$ is a triangle in $P^{\prime}$. By the inductive assumption, $\Delta e v$ contains precisely one point of $S^{\prime}$, and therefore of $S$.

Now suppose $v=v_{0}$. If $e=f_{0}$, then by the way $S$ was constructed, $\Delta e v$ contains the unique point $p_{0}$. Also, if $e=v_{k+1} w_{m+1}$, then $\Delta e v$ contains the unique point $p_{n-3}$. If $e=e_{i}$ for some $1 \leq i \leq k$, then $\Delta e v \cap T_{i}$ contains all chambers $C_{i, j}$ for $i-1 \leq j \leq n-3$. Since $a_{i} \geq i, \Delta e v$ contains $p_{i}$. Also, $\Delta e v \cap T_{i+1}$ contains the chambers $C_{i+1, j}$ for $0 \leq j \leq i-1$. Since $a_{i+1} \geq i+1, p_{i+1}$ does not lie in $\Delta e v$.

Reasoning in a similar fashion, if $1 \leq i \leq m$ and $e=f_{i}$, then $\Delta e v \cap U_{i}$ contains chambers $D_{i, i}$ to $D_{i, n-3}$, and $\Delta e v \cap U_{i+1}$ contains chambers $D_{i+1,0}$ to $D_{i+1, i}$. Since $b_{i} \geq i, S_{i, b_{i}}$ is one of the chambers in $\Delta e v$, and thus $\Delta e v$ contains $p_{i+k}$. Furthermore, $\Delta e v$ does not contain $D_{i+1, b_{i+1}}$, so that $\Delta e v$ does not contain $p_{i+k+1}$.

Finally, suppose $e \in\left\{v_{0} w_{0}, e_{0}\right\}$. We may assume $v \notin\left\{v_{0}, v_{1}, w_{0}\right\}$, for otherwise $\Delta e v$ contains $p_{0}$. By the inductive assumption, $\Delta v_{1} w_{0} v$ contains precisely one point of $S^{\prime}$. In particular, $\Delta v_{1} w_{0} v$ contains $p_{1}$, and no other points of $S^{\prime}$. By the way $S$ was constructed, if $e=v_{0} w_{0}$, then $\Delta e v$ contains $p_{0}$. Furthermore, since $\Delta v_{1} w_{0} v$ contains only $p_{1}$, there are no other points of $S$ in $\Delta e v$. On the other hand, if $e=e_{0}$, then $\Delta e v$ contains $p_{1}$. Since $\Delta v_{1} w_{0} v$ contains no other points of $S$, then neither does $\Delta e v$.

Thus every triangle of the form $\Delta e v$ contains precisely one point of $S$. By Lemma $3, S$ is a pebble set.

Now we show that all peripheral pebble sets are derivable from the construction described above. Let $S$ be any pebble set, all of whose points lie in the periphery of $P$. Suppose the vertices of $P$ are labeled so that $S$ contains a point $p_{0}$ close to $e_{n-1}$. Since all points of $S$ lie in the periphery, there exists precisely one other point $p_{n-3}$ close to some other edge of $P$. Relabel vertices, edges, border triangles, and chambers of the border triangles as was done above, so that $p_{0}$ is close to edge $v_{0} w_{0}$, and $p_{n-3}$ is close to $v_{k+1} w_{m+1}$ for the appropriate $k$ and $m$ such that $k+m=n-4$. Since each border triangle contains precisely one point of $S$, we may let $A$ be the 
set of all positive integers $a_{i}$ for which $S$ contains a point in $C_{i, a_{i}}(1 \leq i \leq k)$, and $B$ the set of all positive integers for which $S$ contains a point in $D_{i, b_{i}}(1 \leq i \leq m)$. We show that $A$ is a uniquely determined subset of $W=\{1,2, \ldots, n-4\}$, and that $B=W-A$.

Since $p_{0}$ lies close to $v_{0} w_{0}$, no other points of $S$ can lie in $T_{0}$ or $U_{0}$. But since $\Delta e_{0} w_{1}$ and $\Delta f_{0} v_{1}$ must contain precisely one point of $S$, then there exists a unique point $p_{1} \in S$ in either $C_{1,1}$ or $D_{1,1}$. Thus either $a_{1}=1 \in A$ or $b_{1}=1 \in B$, but not both.

Now suppose that $s \geq 1$ and that for $1 \leq t \leq s$, either $t \in A$ or $t \in B$, but not both. We may assume that $s \in A$, for a similar argument would work in the event $s \in B$. Let $i$ be the number of elements in $A \cap\{1,2, \ldots, s\}$, so that $a_{i}=s$. Let $j$ be the number of elements in $B \cap\{1,2, \ldots, s\}$, and let $p_{s}$ be the point of $S$ that lies in $C_{i, a_{i}}$.

Notice that for $1 \leq h \leq n-2, \Delta e_{i} v_{i-h}$ (where $i-h$ is taken modulo $n$ ) when intersected with $T_{i}$, yields all chambers $C_{i, h-1}$ to $C_{i, n-3}$. Since $w_{j}=v_{n-1-i}=$ $v_{i-(i+j+1-n)}$, we may let $h=i+j+1$ to have that $\Delta e_{i} w_{j}$ intersects $T_{i}$ to yield the chambers from $C_{i, i+j}=C_{i, s}$ to $C_{i, n-3}$. Thus $p_{s}$ is the unique point of $S$ in $\Delta e_{i} w_{j}$. Similarly, $\Delta e_{i+1} w_{j}$ intersects $T_{i+1}$ to yield $C_{i+1,0}$ through $C_{i+1, i+j+1}$. Thus $\Delta e_{i} w_{j}$ intersects $T_{i+1}$ to yield $C_{i+1,0}$ through $C_{i+1, i+j}$. Thus in $T_{i+1}$ there are no points of $S$ in any of the $C_{i+1, h}$ for $0 \leq h \leq i+j$. But $\Delta e_{i} v_{j+1}$ contains a point of $S$. Thus there must exist a point $p_{s+1}$ either in $C_{i+1, i+j+1}$ or in $D_{j+1, i+j+1}$, but not both. Since $i+j=s$, there exists a point of $S$ in either $C_{i+1, s+1}$ or $D_{j+1, s+1}$. If $p_{s+1} \in C_{i+1, s+1}$, then $s+1 \in A$, and we may write $a_{i+1}=s+1$. On the other hand, if $p_{s+1} \in D_{j+1, s+1}$, then $s+1 \in B$, and we may write $b_{j+1}=s+1$.

By induction $(1 \leq s \leq n-4), A$ and $B$ are uniquely determined.

If $n=3$, then an $n$-gon has a unique pebble set. If $n \geq 4$, then the power set of $\{1,2, \ldots, n-4\}$ contains $2^{n-4}$ elements. Thus we have the following.

Corollary 5 Let $P$ be a convex $n$-gon, and $e$ an edge of $P$. Let $d(P)=1$ if $n=3$, and $d(P)=0$ otherwise. Then the number of peripheral pebble sets in $P$ that contain a point close to $e$ is $2^{n-4+d(P)}$.

If $n \geq 4$, we may sum the numbers of pebble sets from Corollary 5 across all edges of an $n$-gon. Since a peripheral pebble set contains precisely two points close to an edge, we count each pebble set exactly twice. Thus we arrive at the following.

Corollary 6 If $P$ is an $n$-gon $(n \geq 4)$, then the number of peripheral pebble sets is $n 2^{n-5}$.

\section{Characterizing Non-Peripheral Pebble Sets}

For a given $n$-gon, pebble sets other than peripheral pebble sets might exist. In this section, we want to characterize chambers for which a pebble set exists that contains a point in the chamber. An important example of such a chamber lies in the hexagon in Fig. 4. 
Fig. 4 Example of a non-peripheral pebble set in a hexagon

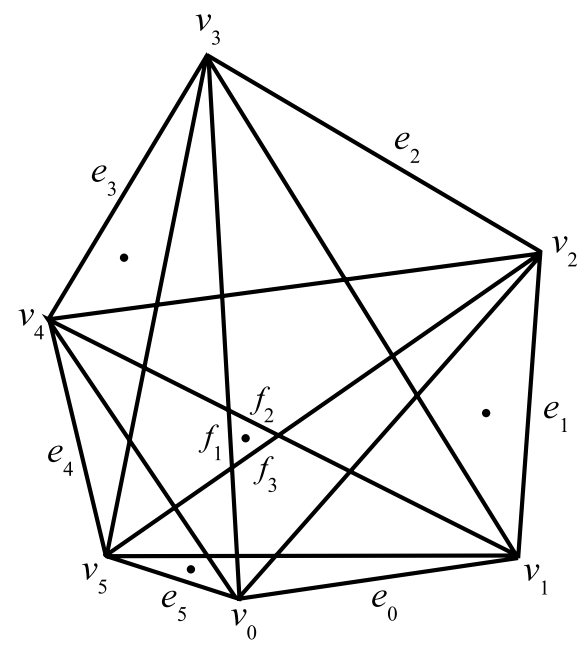

In any hexagon $H$, the chords $f_{1}=v_{0} v_{3}, f_{2}=v_{1} v_{4}$, and $f_{3}=v_{2} v_{5}$ intersect pairwise. If these three points of intersection are distinct, they determine a triangular region $R$ in $H$. For any given border triangle $T$, none of these three points lies in $T$, so that $R$ lies in the core of $H$. Furthermore, all chords of $H$ other than $\left\{f_{1}, f_{2}, f_{3}\right\}$ are boundary segments of the border triangles, and thus do not intersect the core of $H$. Therefore, $R$ is a triangular chamber in the core of $H$.

\subsection{Edge Supports and Viability}

Definition 3 We say that a chamber is viable if there exists a pebble set containing a point in the chamber.

By Theorem 4, we see that every chamber in the periphery of a polygon is viable.

Definition 4 Given a chamber $C$ of a polygon $P$, the edge support of $C$, written $\mathrm{ES}(C)$, is the set of edges $e$ of $P$ for which $C$ is contained in the fan of $e$.

As an example, note that in Fig. 4, the edge support of the triangular chamber in the core is $\left\{e_{0}, e_{2}, e_{4}\right\}$. By the support size of $C$, we mean the cardinality of $\operatorname{ES}(C)$, which we denote $|\operatorname{ES}(C)|$. A major result of this section is that a chamber $C$ is viable if and only if $|\operatorname{ES}(C)|=3$ (Theorem 17 and Corollary 22). Along the way, we note other necessary conditions of viability that are helpful in practice. In particular, if the edge support of a viable chamber contains two incident edges, the chamber must lie in the periphery (Proposition 15). In addition, the only pebble sets in a regular polygon are peripheral (Theorem 16).

Proposition 7 If $C$ is a chamber in a polygon and $v_{k}$ is any vertex, then there exists a unique edge $e_{j}(j \notin\{k-1, k\})$ such that $C$ is contained in $\Delta e_{j} v_{k}$. 
Fig. 5 A possible chamber from Proposition 9

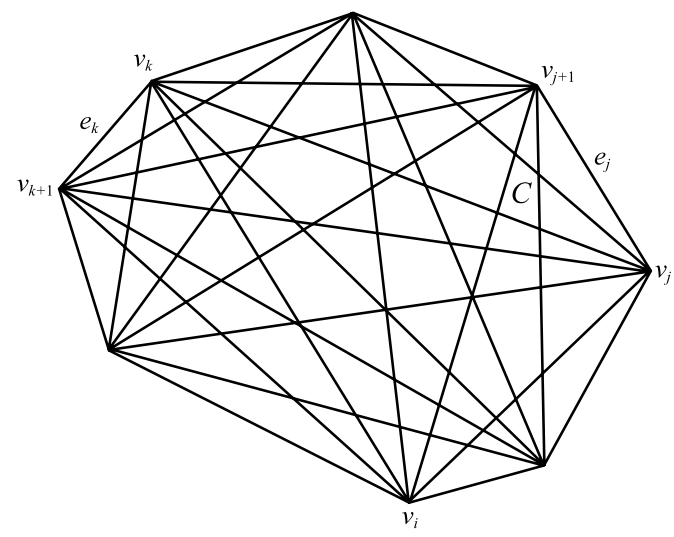

Proof The chords of the polygon emanating from $v_{k}$ form a triangulation of the polygon. Thus $C$ must lie within precisely one of these triangles, which are of the form $\Delta e_{j} v_{k}$, where $j \notin\{k-1, k\}$.

Proposition 8 If $C$ is a chamber in the periphery of a polygon, then $|\mathrm{ES}(C)|=3$, and at least two edges of $\mathrm{ES}(C)$ are incident.

Proof Suppose $C$ is in the periphery. Then it is contained in the border triangle at some $v_{k}$, so that $e_{k-1}, e_{k} \in \operatorname{ES}(C)$. Applying Proposition 7 to $v_{k}$, we see that there is an additional edge in $e_{j} \in \mathrm{ES}(C)$. Now any triangle containing $C$ must include $v_{k}$ as a vertex. Since $e_{j}$ is the unique edge such that $C$ is contained in $\Delta e_{j} v_{k}$, there are no other edges in $\operatorname{ES}(C)$.

For convenience in the next proof, we will say that a vertex $v_{j}$ is between vertices $v_{i}$ and $v_{k}$ if the counterclockwise ordering of the three vertices is $\left\langle v_{i}, v_{j}, v_{k}\right\rangle$.

Proposition 9 Suppose $v_{k}, v_{k+1}, v_{j}$ and $v_{j+1}$ are distinct vertices in a polygon, and that $C$ is a chamber in $\Delta e_{j} v_{k}$. Let $v_{i}$ be a vertex between $v_{k}$ and $v_{j}$, and suppose also that $C$ lies in $\Delta e_{j} v_{i}$. Then $C$ is not in the fan of $e_{k}$. (See Fig. 5 for an illustration.)

Proof Let $v_{m}$ be any vertex $(m \notin\{k, k+1\})$. We show that $C$ is not in $\Delta e_{k} v_{m}$ to have that $C$ is not in the fan of $e_{k}$. If $v_{m}$ is between $v_{k+1}$ and $v_{j+1}$, then we may triangulate the polygon by including the chord from $v_{k+1}$ to $v_{m}$, all chords from $v_{k+1}$ to the vertices between $v_{k+1}$ and $v_{m}$, the chord from $v_{k}$ to $v_{m}$, and all chords from $v_{k}$ to the remaining vertices. This triangulation contains $\Delta e_{k} v_{m}$ and $\Delta e_{j} v_{k}$, and are therefore disjoint. Since $C$ is contained in $\Delta e_{j} v_{k}$ it is not in $\Delta e_{k} v_{m}$.

If $v_{m}$ is between $v_{j}$ and $v_{k}$, triangulate the polygon by including chords from $v_{i}$ to all vertices between $v_{i}$ and $v_{m}$, and chords from $v_{m}$ to all remaining vertices. This triangulation contains $\Delta e_{j} v_{i}$ and $\Delta e_{k} v_{m}$. Since $C$ is in $\Delta e_{j} v_{i}$ it is not in $\Delta e_{k} v_{m}$.

In either case, $C$ is not contained in $\Delta e_{k} v_{m}$. Since this is true for all $m$, we have that $C$ is not in the fan of $e_{k}$. 
To characterize viable chambers in the core of a polygon, we will need to relate a given $n$-gon to an $(n-1)$-gon formed by removing a vertex, as in the proof of Theorem 4.

Let $v_{k}$ be a vertex in an $n$-gon $P$, and let $C$ be a chamber of $P$ not in the border triangle at $v_{k}$. Let $P^{\prime}$ be the $(n-1)$-gon formed by deleting $v_{k}$ and including the edge $v_{k-1} v_{k+1}$, and let $C^{\prime}$ be the chamber of $P^{\prime}$ that contains $C$. Since chambers do not intersect any chord in a polygon, we may make the following observation.

Proposition 10 Suppose $T$ is a triangle determined by three vertices of $P^{\prime}$, and suppose $T$ contains $C$. Then $T$ also contains $C^{\prime}$.

Proposition 10 allows us to show that an edge $e$ is in $\operatorname{ES}\left(C^{\prime}\right)$ by showing merely that $C$ is contained in $\Delta e v$ for some vertex $v$ in $P^{\prime}$. The following proposition relates the edge support of a chamber in $P$ to the edge support of the chamber that contains it in $P^{\prime}$.

Proposition 11 Let $v_{k}$ be a vertex in an $n$-gon $P$, let $C$ be a chamber of $P$ not in the border triangle at $v_{k}$, and let $C$ have edge support $\operatorname{ES}(C)$. Let $P^{\prime}$ be the $(n-1)$-gon formed by deleting $v_{k}$ and including the edge $f=v_{k-1} v_{k+1}$. Let $C^{\prime}$ be the chamber of $P^{\prime}$ that contains $C$, and let $\operatorname{ES}\left(C^{\prime}\right)$ be the edge support of $C^{\prime}$ in $P^{\prime}$. Then the following are true:

1. If neither $e_{k-1}$ and $e_{k}$ is in $\operatorname{ES}(C)$, then $\operatorname{ES}\left(C^{\prime}\right)=\operatorname{ES}(C)$.

2. If precisely one of $e_{k-1}$ and $e_{k}$ is in $\operatorname{ES}(C)\left(\right.$ say $\left.e_{k}\right)$, then $\operatorname{ES}\left(C^{\prime}\right)=[\operatorname{ES}(C)-$ $\left.\left\{e_{k}\right\}\right] \cup\{f\}$.

3. If $e_{k-1}, e_{k} \in \mathrm{ES}(C)$, then there exists an edge $e_{j} \in \operatorname{ES}(C)(j \notin\{k-1, k\})$ such that $\mathrm{ES}\left(C^{\prime}\right)=\left[\mathrm{ES}(C)-\left\{e_{k-1}, e_{k}, e_{j}\right\}\right] \cup\{f\}$.

Proof We prove the result by showing the following.

1. $f \in \operatorname{ES}\left(C^{\prime}\right)$ if and only if $e_{k-1} \in \operatorname{ES}(C)$ or $e_{k} \in \operatorname{ES}(C)$.

2. For every other edge $e_{j}$ in $P^{\prime}$ (so that $j \notin\{k-1, k\}$ ), the following hold:

(a) If $e_{k-1}$ and $e_{k}$ are not both in $\operatorname{ES}(C)$, then $e_{j} \in \operatorname{ES}(C)$ if and only if $e_{j} \in$ $\mathrm{ES}\left(C^{\prime}\right) ;$ and

(b) If $e_{k-1}, e_{k} \in \operatorname{ES}(C)$, and if (by Proposition 7) $e_{j}$ is the unique edge for which $C$ is contained in $\Delta e_{j} v_{k}$, then $e_{j} \in \operatorname{ES}(C)-\operatorname{ES}\left(C^{\prime}\right)$, and for every other edge $e_{i}(i \neq j), e_{i} \in \operatorname{ES}\left(C^{\prime}\right)$ if and only if $e_{i} \in \operatorname{ES}(C)$.

For claim 1, if $f \in \operatorname{ES}\left(C^{\prime}\right)$, then there exists a vertex $v_{j}(j \notin\{k-1, k, k+1\})$ such that $C^{\prime}$ is contained in $\Delta f v_{j}$. This triangle is itself contained in the union of $\Delta e_{k-1} v_{j}$ and $\Delta e_{k} v_{j}$. Thus $C$ is contained in one of these triangles, so that either $e_{k-1}$ or $e_{k}$ is in $\operatorname{ES}(C)$. Conversely, if either $e_{k-1}$ or $e_{k}$ is in $\operatorname{ES}(C)$, then we may consider that $e_{k} \in \operatorname{ES}(C)$ (the argument for $e_{k-1}$ would be similar). Thus there exists a vertex $v_{j}$ $(j \notin\{k, k+1\})$ such that $C$ is contained in $\Delta e_{k} v_{j}$. But $j \neq k-1$ also, because $C$ is not in the border triangle at $v_{k}$. Thus $C$ is contained in $\Delta f v_{j}$. By Proposition 10, $C^{\prime}$ is also contained in $\Delta f v_{j}$, so that $f \in \operatorname{ES}\left(C^{\prime}\right)$.

For claim 2a, suppose $j \notin\{k-1, k\}$ and $e_{k} \notin \mathrm{ES}(C)$. (The arguments for the case of $e_{k-1} \notin \operatorname{ES}(C)$ would be similar to the ones to follow.) Suppose $e_{j} \in \operatorname{ES}(C)$ and 
that $e_{j} \notin \mathrm{ES}\left(C^{\prime}\right)$. Then there exists a vertex $v_{i}$ such that $C$ is contained in $\Delta e_{j} v_{i}$. If $i \neq k$, then $\Delta e_{j} v_{i}$ contains $C$, and therefore contains $C^{\prime}$ by Proposition 10. This contradicts the fact that $e_{j} \notin \mathrm{ES}\left(C^{\prime}\right)$. Therefore, $v_{k}$ is the only vertex such that $C$ is contained in $\Delta e_{j} v_{k}$. Since $e_{k} \notin \mathrm{ES}(C)$, it is impossible that $j=k+1$. Thus the edges $e_{j}$ and $e_{k}$ determine a quadrilateral in $P$, which is the union of triangles $\Delta e_{k} v_{j+1}$ and $\Delta e_{j} v_{k+1}$. Furthermore, $C$ must lie in this quadrilateral, because it lies in $\Delta e_{j} v_{k}$. But $C$ does not lie in $\Delta e_{k} v_{j}$ because $e_{k} \notin \mathrm{ES}(C)$. Also, $C^{\prime}$ does not lie in $\Delta e_{j} v_{k+1}$ by supposition, so that $C$ does not lie in $\Delta e_{j} v_{k+1}$ either. This is a contradiction, so it must be that $e_{j} \in \mathrm{ES}\left(C^{\prime}\right)$.

Conversely, suppose $e_{j} \in \operatorname{ES}\left(C^{\prime}\right)$. Then there exists vertex $v_{i}(i \neq k)$ such that $C^{\prime}$ is contained in $\Delta e_{j} v_{i}$. Since $C \subseteq C^{\prime}, C$ is also contained in $\Delta e_{j} v_{i}$, so that $e_{j} \in$ $\mathrm{ES}(C)$.

For claim 2b, suppose $e_{k-1}, e_{k} \in \operatorname{ES}(C)$ and that $e_{j}$ is the unique edge such that $C$ is contained in $\Delta e_{j} v_{k}$. Then clearly $e_{j} \in \operatorname{ES}(C)$, so we must show that $e_{j} \notin \operatorname{ES}\left(C^{\prime}\right)$. First suppose that $j=k+1$. Then $C$ is in the border triangle at $v_{k+1}$. By Proposition $8, \operatorname{ES}(C)=\left\{e_{k-1}, e_{k}, e_{k+1}\right\}$, and by Proposition 7 applied to $v_{k+1}, C$ must lie in the border triangle at $v_{k}$. This is impossible, since $C$ is assumed not to be in this border triangle. By similar reasoning, $j \neq k-2$. Thus $e_{k-1}, e_{k}$ and $e_{j}$ determine a pentagon in $P$, and we may apply Proposition 9. If $C$ is contained in $\Delta e_{j} v_{i}$ for some vertex $v_{i}$ between $v_{k}$ and $v_{j}$, then $C$ cannot be in the fan of $e_{k}$. But $e_{k} \in \operatorname{ES}(C)$ by assumption. Reasoning similarly from Proposition 9, if $C$ is contained in $\Delta e_{j} v_{i}$ for some $v_{i}$ between $v_{j+1}$ and $v_{k+1}$, then $C$ cannot be in the fan of $e_{k-1}$. But $e_{k-1} \in \operatorname{ES}(C)$. Thus for any $i \neq k, C$ does not lie in $\Delta e_{j} v_{i}$, and neither is $C^{\prime}$ contained in $\Delta e_{j} v_{i}$. Therefore, $e_{j} \notin \mathrm{ES}\left(C^{\prime}\right)$.

Finally, if $e_{i}$ is an edge, $i \neq j$, we show that $e_{i} \in \operatorname{ES}\left(C^{\prime}\right)$ if and only if $e_{i} \in \operatorname{ES}(C)$. As before, if $e_{i} \in \operatorname{ES}\left(C^{\prime}\right)$, then there exists a vertex $v_{m}$ so that $C^{\prime} \subset \Delta e_{i} v_{m}$. Since $C^{\prime} \supset C$, we have that $e_{i} \in \operatorname{ES}(C)$. Conversely, suppose $e_{i} \in \operatorname{ES}(C)$. Then there exists a vertex $v_{m}$ so that $C \subset \Delta e_{i} v_{m}$. Now $m$ cannot equal $k$, since $i \neq j$ and $\Delta e_{j} v_{k}$ is the unique triangle given by Proposition 7 based at $v_{k}$ containing $C$. Therefore, $\Delta e_{i} v_{m}$ is in $P^{\prime}$, and so $e_{i} \in \operatorname{ES}\left(C^{\prime}\right)$.

Proposition 11 implies the following about the support size of $C$, and its relationship to the support size of $C^{\prime}$.

Corollary 12 Let $C$ be a chamber in an n-gon $P$. Then $|\mathrm{ES}(C)|$ is odd and at least three. In fact, If $v_{k}$ is a vertex whose border triangle does not contain $C$, and $C^{\prime}$ is the chamber in the $(n-1)$-gon created from $P$ by deleting $v_{k}$, then $|\operatorname{ES}(C)|=$ $\left|\mathrm{ES}\left(C^{\prime}\right)\right|+2$ whenever $e_{k-1}, e_{k} \in \operatorname{ES}(C)$. Otherwise, $|\mathrm{ES}(C)|=\left|\operatorname{ES}\left(C^{\prime}\right)\right|$.

Proof If $n=3$ then $|\operatorname{ES}(C)|=3$. So suppose $n \geq 4$ and that the result is true for all polygons on fewer than $n$ vertices. Now $C$ cannot be in every border triangle. We may therefore remove some vertex $v_{k}$ whose border triangle does not contain $C$ to create the $n$-gon $P^{\prime}$ with a chamber $C^{\prime} \supseteq C$, where by the inductive assumption, $\left|\operatorname{ES}\left(C^{\prime}\right)\right|$ is odd and at least three. If $e_{k-1}, e_{k} \in \operatorname{ES}(C)$, then by Proposition 11, $|\operatorname{ES}(C)|=$ $\left|\mathrm{ES}\left(C^{\prime}\right)\right|+2$. Otherwise, $|\mathrm{ES}(C)|=\left|\mathrm{ES}\left(C^{\prime}\right)\right|$.

We will show that a support size of three is necessary and sufficient for the viability of a chamber. First we show the following. 
Proposition 13 Suppose $S$ is a pebble set in an $n$-gon $P(n \geq 4)$, and $v$ is any vertex of $P$. Let $p$ be the point of $S$ that lies in the border triangle at $v$, and let $P^{\prime}$ be the polygon formed from $P$ by removing $v$. Then $S-\{p\}$ is a pebble set in $P^{\prime}$.

Proof If $T$ is any triangle in $P^{\prime}$, it is also a triangle in $P$. Thus $T$ contains a unique point of $S$. Now every triangle that contains $p$ includes $v$ as a vertex, so that $p$ is not the point of $S$ that lies in $T$. Therefore, $S-\{p\}$ is a pebble set in $P^{\prime}$.

Corollary 14 Suppose $C$ is a viable chamber in an $n$-gon $(n \geq 4)$, and that $C$ is not in the border triangle at vertex $v$. Then $C^{\prime} \supseteq C$ is viable in the $(n-1)$-gon created by removing $v$.

\subsection{Proof of Necessity}

We are now ready to show that every viable chamber has support size three. In Sect. 4.3 we will prove the converse. In preparation for the proof of necessity, we first demonstrate another necessary condition for viability that has practical application. Proposition 15 implies that if a chamber lies in the core of a polygon and its edge support contains two incident edges, then it is not viable.

Proposition 15 If $C$ is a viable chamber and $\operatorname{ES}(C)$ contains two incident edges, then $C$ is in the periphery.

Proof Suppose $C$ is viable and $E S(C)$ contains $e_{k}$ and $e_{k+1}$. Then there exist vertices $v_{i}, v_{j}(i, j \neq k+1)$ such that $\Delta e_{k} v_{i}$ and $\Delta e_{k+1} v_{j}$ contain $C$. Let $T$ be the border triangle at $v_{k+1}$. Now $T$ consists of $n-2$ chambers, which we label $C_{0}, \ldots, C_{n-3}$, where $C_{0}=T \cap \Delta e_{k-1} v_{k+1}$, and the others are numbered consecutively up to $C_{n-3}=$ $T \cap \Delta e_{k+2} v_{k+1}$.

By Proposition 7, there is an edge $e_{m}$ so that $\Delta e_{m} v_{k+1}$ contains $C$. Thus $T \cap \Delta e_{m} v_{k+1}$ is a single chamber, which we call $C_{m}$. By convexity, any triangle containing $C$ and $v_{k+1}$ must intersect (and therefore contain) $C_{m}$.

Since the triangles $\Delta e_{k} v_{i}$ and $\Delta e_{k+1} v_{j}$ both contain $C$, they both contain $C_{m}$. Now $\Delta e_{k+1} v_{j}$ contains $C_{0}$, so by convexity, it contains chambers $C_{0}$ through $C_{m}$. Similarly, $\Delta e_{k} v_{i}$ contains $C_{m}$ and $C_{n-3}$, and therefore, all the chambers $C_{m}$ through $C_{n-3}$. We can then conclude that $\Delta e_{k} v_{i} \cup \Delta e_{k+1} v_{j}$ contains $T$.

Now since $\Delta e_{k} v_{i}$ is a triangle, any pebble set containing a point in $C$ cannot contain another point in $\Delta e_{k} v_{i}$. Similarly for $\Delta e_{k+1} v_{j}$. Thus a pebble set containing a point in $C$ can have no other point in $T$. But $T$ is a triangle, and so the pebble set must have a point in it. Therefore $C$ must be in $T$, and hence in the periphery.

An interesting implication of Proposition 15 is the following.

Theorem 16 If $P$ is a regular $n$-gon, then all pebble sets are peripheral.

Proof If $n \in\{3,4\}$ then every chamber is in the periphery, so let $n \geq 5$, and let $P$ be the regular polygon on $n$ vertices. We show that every point in the polygon that does not lie on a chord is in a chamber whose edge support contains two incident 
Fig. 6 A regular polygon from the proof of Theorem 16

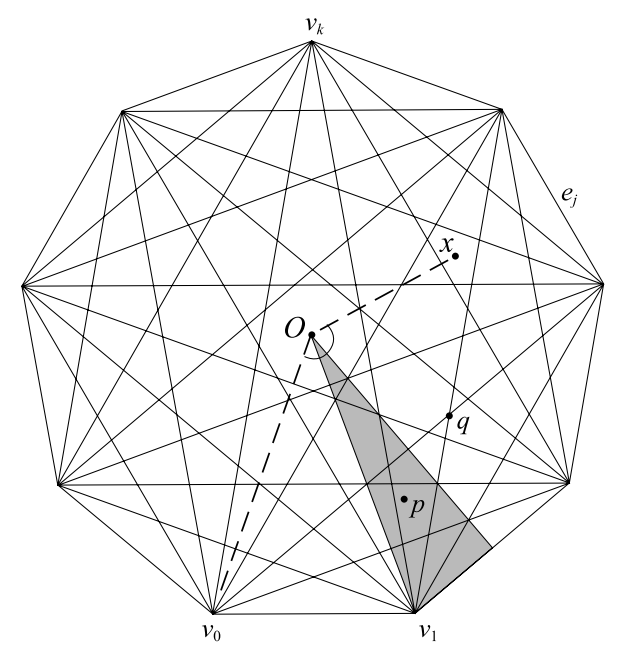

edges. Let $O$ be the center point of $P$. For a point $x \neq O$ anywhere in $P$, define $\theta(x)$ to be the counterclockwise angle between segments $O v_{0}$ and $O x(0 \leq \theta<2 \pi)$. Let $k=\lceil n / 2\rceil$. Then $k$ is the smallest positive integer for which $\theta\left(v_{k}\right) \geq \pi$.

Suppose $p$ is any point in a viable chamber $C$ of $P$. Since chambers are open and nonempty, we may assume $p \neq O$. By rotating the indices of $P$ and exploiting reflective symmetries of $P$, we may assume that $\frac{2 \pi}{n} \leq \theta(p) \leq \frac{3}{2} \frac{2 \pi}{n}$. In other words, we may assume that $p$ lies in a closed triangular $B \subseteq P$ defined by $O, v_{1}$, and the midpoint of $e_{1}$.

If $p$ lies in $\Delta e_{1} v_{0}$, then it is in the periphery. So we may assume otherwise. By the way $k$ was chosen, we may consider a triangulation of chords emanating from $v_{0}$ and have that there is some $e_{j}(2 \leq j \leq k-1)$ such that $p$ lies in $\Delta e_{j} v_{0}$. This triangle is a subset of the quadrilateral determined by $e_{0}$ and $e_{j}$. We show that $p$ is in the fans of both $e_{0}$ and $e_{1}$.

First we show that $p$ is in the fan of $e_{1}$. If $n$ is odd, then $\Delta e_{1} v_{k+1}$ contains $O$, so that it contains all of $B$. Thus $p$ lies in $\Delta e_{1} v_{k+1}$. If $n$ is even, then chord $v_{1} v_{k+1}$ passes through $O$. Since $p$ does not lie on a chord of $P$, then $p$ must lie in $\Delta e_{1} v_{k+1}$. In either case, $p$, and therefore all of $C$, lies in the fan of $e_{1}$.

Now we show that $p$ is in the fan of $e_{0}$. Suppose that $p$ does not lie in either $\Delta e_{0} v_{j}$ or $\Delta e_{0} v_{j+1}$. Then it must lie in $\Delta e_{j} v_{1} \cap \Delta e_{j} v_{0}$. Now the chords $v_{0} v_{j}$ and $v_{1} v_{j+1}$ intersect at a point $q$ where $\theta(q)$ is the average of $\theta\left(v_{1}\right)=2 \pi / n$ and $\theta\left(v_{j}\right)=2 j \pi / n$. Thus $\theta(q)=(j+1) \pi / n$, so that $\theta(p)>\pi(j+1) / n$. But since $j \geq 2$, we have that $\theta(p) \geq 3 \pi / n$. This is a contradiction, so $p$ must lie in either $\Delta e_{0} v_{j}$ or $\Delta e_{0} v_{j+1}$, Thus $p$, and therefore $C$, lies in the fan of $e_{0}$.

Therefore $e_{0}$ and $e_{1}$, which are incident edges, are in $E S(C)$, and by Proposition 15 , we can conclude $C$ is in the periphery.

Theorem 16 can actually be stated in a slightly stronger form. If $P$ is an $n$-gon for which the intersections of the chords generates the same internal topology as the regular $n$-gon, then all pebble sets are peripheral. Given that we have characterized all peripheral pebble sets in general in Sect. 3, we therefore have solved the problem 
of characterizing all pebble sets for polygons whose internal topology is equivalent to that of a regular polygon.

We are now ready to prove one direction of the viability question.

Theorem 17 If $C$ is a viable chamber in an $n$-gon, then $|\operatorname{ES}(C)|=3$.

Proof Suppose $C$ is viable. If $n=3$, the result it clear, so suppose $n \geq 4$, and that the result is true for all polygons on fewer than $n$ vertices. If $C$ is in the periphery, then $|\operatorname{ES}(C)|=3$ by Proposition 8 . Thus we may assume $C$ is in the core, so that $\mathrm{ES}(C)$ does not contain two incident edges, by Proposition 15. Let $v_{k}$ be a vertex for which $e_{k-1}$ and $e_{k}$ are not both in $\operatorname{ES}(C)$. Remove $v_{k}$ to form the $(n-1)$-gon as in Proposition 11. Since at least one of $e_{k}$ and $e_{k-1}$ is not in $\operatorname{ES}(C)$, then by Corollary $12,|\mathrm{ES}(C)|=\left|\mathrm{ES}\left(C^{\prime}\right)\right|$. By Corollary $14, C^{\prime}$ is viable, so that by the inductive assumption, $\left|\mathrm{ES}\left(C^{\prime}\right)\right|=3$. Thus $|\mathrm{ES}(C)|=3$.

An important consequence of Theorem 17 is the following.

Proposition 18 A chamber with support size three is triangular.

Proof Suppose $|\mathrm{ES}(C)|=3$. Since all chambers in the periphery are triangular, we assume $C$ is in the core. We demonstrate a one-to-one correspondence between the chords that determine the boundary of $C$ and the pairs of edges chosen from $\operatorname{ES}(C)$. Let $v_{i} v_{j}$ be a chord that determines part of the boundary of $C$. The line $\overleftrightarrow{v_{i} v_{j}}$ cuts the plane into two half planes. We may assume that $C$ lies in the half plane containing edges $e_{i-1}$ and $e_{j}$. Since $C$ is a chamber, it lies in both $\Delta e_{i-1} v_{j}$ and $\Delta e_{j} v_{i}$. Thus $e_{i-1}, e_{j} \in \mathrm{ES}(C)$, and we have that every chord $v_{i} v_{j}$ of $P$ determines a unique pair of edges in $\operatorname{ES}(C)$ that lie in the same half plane as $C$ upon cutting the plane with $v_{i} v_{j}$.

Now let $v_{s} v_{t}$ be a chord of $P$ that determines part of the boundary of $C$. We show that the only way the above algorithm can yield $e_{i-1}$ and $e_{j}$ is if $v_{s} v_{t}=v_{i} v_{j}$. Since $C$ lies in the core, there are four distinct chords incident with $e_{i-1}$ and $e_{j}$, defined by the four distinct endpoints of $e_{i-1}$ and $e_{j}$. Since the chord $v_{i-1} v_{j}$ cuts the plane so that $e_{i-1}$ and $e_{j}$ lie in different half planes, it is impossible that $v_{s} v_{t}=v_{i-1} v_{j}$. By similar reasoning $v_{s} v_{t} \neq v_{i} v_{j+1}$. Furthermore, since $C$ is a chamber, it is impossible that the chord $v_{i-1} v_{j+1}$ form part of the boundary of $C$, since $v_{i} v_{j}$ and $v_{i-1} v_{j+1}$ lie on opposite sides of $v_{i} v_{j+1}$. Thus the only chord for which the above algorithm can determine $e_{i-1}$ and $e_{j}$ is $v_{i} v_{j}$.

\subsection{Proof of Sufficiency}

Now we address the converse of Theorem 17 by showing that if the support size of a chamber is three, then the chamber is viable. We will use the three edges in its edge support to demonstrate a construction that produces all pebble sets containing a point in the chamber. We begin by describing a construction whereby such a chamber can be used to determine three polygonal subsets of a given $n$-gon.

Construction 2 Let $C$ be a chamber in an $n$-gon $P$ with support size three, and let the edges in $\operatorname{ES}(C)$ be labeled counterclockwise as $e_{1}, e_{2}$, and $e_{3}$. By Proposition 18, 
Fig. 7 A triangular chamber with support size three

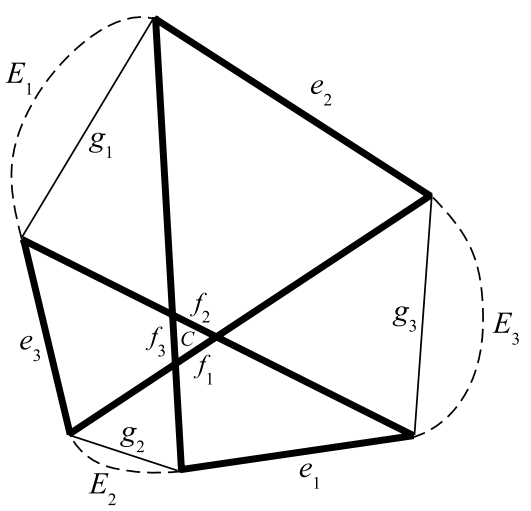

$C$ is triangular. (See Fig. 7.) Label the three chords that define the boundary of $C$ as $f_{1}, f_{2}$, and $f_{3}$, where $f_{i}$ is incident with $e_{j}$ and $e_{k}(i, j$, and $k$ all distinct). Similarly, for $1 \leq i \leq 3$, let $E_{i}$ be the set of edges of $P$ between $e_{j}$ and $e_{k}(i, j$, and $k$ all distinct). Note that some of the $E_{i}$ might be empty. Let $Q_{1}$ be the polygonal subset of $P$ whose perimeter is defined by $e_{3}, f_{1}, e_{2}$, and all edges in $E_{1}$. Let $Q_{2}$ and $Q_{3}$ be analogously defined polygonal subsets of $P$. Finally, let $p$ be any point in $C$.

Since $C$ is not in the fan (in $P$ ) of any edge in $E_{1}$, it is also not in the fan in $Q_{1}$ of any edge in $E_{1}$. Thus the edge support of $C$ in $Q_{1}$ is precisely $\left\{f_{1}, e_{2}, e_{3}\right\}$, and $p$ lies close to $f_{1}$ in $Q_{1}$. By Theorem 4 , there exists a pebble set $S_{1}$ in $Q_{1}$ that includes $p$. By similar reasoning, there exist pebble sets $S_{2}$ and $S_{3}$ in $Q_{2}$ and $Q_{3}$, respectively, that include $p$. Let $S=S_{1} \cup S_{2} \cup S_{3}$.

We now show that $S$ as described in Construction 2 is a pebble set in $P$ that contains $p$, and that all pebble sets in $P$ that contain $p$ are uniquely described in this way. We construct a bijection between the set of all possible ordered triples $\left(S_{1}, S_{2}, S_{3}\right)$ of pebble sets in the $Q_{i}$, where each $S_{i}$ contains $p$, and the set of all pebble sets in $P$ that contain $p$. For a polygon $P$ and a point $p$ in a chamber of $P$, we write $\mathcal{S}(P, p)$ to mean the set of all pebble sets in $P$ that contain the point $p$. Thus the construction we desire is a one-to-one correspondence between $\mathcal{S}(P, p)$ and $\prod_{i=1}^{3} \mathcal{S}\left(Q_{i}, p\right)$.

Let $P$ be a polygon and $S \in \mathcal{S}(P, p)$. Define $\pi: \mathcal{S}(P, p) \rightarrow \prod_{i=1}^{3} \mathcal{S}\left(Q_{i}, p\right)$ to map $S$ to the ordered triple $\left(S \cap Q_{1}, S \cap Q_{2}, S \cap Q_{3}\right)$. To show that $\pi$ is a bijection, the following lemmas will be helpful. For convenience, we state them with specific reference to $Q_{1}$, though analogous results clearly hold for $Q_{2}$ and $Q_{3}$.

Lemma 19 Let $P$ be a polygon and $C$ a chamber of $P$. Let $Q_{1}, E_{2}$ and $E_{3}$ be defined as in Construction 2. Let $S_{1}$ be a pebble set in $Q_{1}$ that contains $p$, and let $D$ be any chamber of $Q_{1}$ that contains a point of $S_{1}-\{p\}$. Then no point in $S_{1}-\{p\}$ lies in the fan of any edge of $E_{2} \cup E_{3} \cup\left\{e_{1}\right\}$.

Proof Let $q$ be any point in $S_{1}-\{p\}$, let $e$ be any edge in $E_{2} \cup E_{3} \cup\left\{e_{1}\right\}$, and let $v$ be any vertex of $P$. If $v$ is not an endpoint of an edge in $E_{1}$, then $\Delta e v$ does not lie in $Q_{1}$, so that $q \notin \Delta e v$. So suppose $v$ is an endpoint of an edge in $E_{1}$. Now $\Delta e v \cap Q_{1} \subset \Delta f_{1} v$, and $q \in Q_{1}$, so if $q \in \Delta e v$, then we would have $q \in \Delta f_{1} v$. Yet, $p \in \Delta f_{1} v$, and $S_{1}$ is a pebble set in $Q_{1}$, and so $q \notin \Delta e v$. 
Lemma 20 Let $P$ be a polygon and $C$ a chamber of $P$. Let $Q_{1}, E_{2}$ and $E_{3}$ be defined as in Construction 2. Let $S_{1}$ be a pebble set in $Q_{1}$ that contains $p$, and let $D$ be any chamber of $Q_{1}$ that contains a point of $S_{1}-\{p\}$. Then $D$ is also a chamber of $P$.

Proof Suppose $D$ is a chamber of $Q_{1}$ that contains some $q \in S_{1}-\{p\}$, and which is not a chamber of $P$. Then there is a chord of $P$ that intersects $D$. Such a chord is clearly not a chord of $Q_{1}$, and is thus of the form $u v$, where $u$ is a vertex of $Q_{1}$ and $v$ is not. Of all such chords, let $u v$ be closest to $q$. If $q$ does not lie on $u v$, then there is an edge $e \in E_{2} \cup E_{3} \cup\left\{e_{1}\right\}$ that is incident with $v$ such that $q \in \Delta e u$. But then $q$ lies in the fan of $e$, which contradicts Lemma 19. If, on the other hand, $q$ lies on $u v$, then the fact that $u v$ intersects $f_{1}$ reveals that $q \in \Delta f_{1} u$, which is impossible. Thus the chords that determine the chamber in $P$ that contains $q$ are also chords of $Q_{1}$, and $D$ is a chamber of $P$.

Theorem 21 The mapping $\pi: \mathcal{S}(P, p) \rightarrow \prod_{i=1}^{3} \mathcal{S}\left(Q_{i}, p\right)$ defined above is a bijection.

Proof First we show that $\pi$ is a function. If $S \in \mathcal{S}(P, p)$, then clearly for $1 \leq i \leq 3$, $S \cap Q_{i}$ is a pebble set in $Q_{i}$ that contains $p$. Thus $\pi(S) \in \prod_{i=1}^{3} \mathcal{S}\left(Q_{i}, p\right)$. To show that $\pi$ is well defined, suppose $S, T \in \mathcal{S}(P, p)$ are equivalent pebble sets. Then the chambers of $P$ that contain the points of $S$ are precisely those that contain the points of $T$. Let $C$ be any such chamber, and suppose $q \in S \cap C$ and $r \in T \cap C$. Suppose also that $C \subseteq Q_{i}$. Since every chamber of $P$ is a subset of some chamber of $Q_{i}$, the chamber in $Q_{i}$ that contains $q$ also contains $r$, so that $S \cap Q_{i}$ and $T \cap Q_{i}$ are equivalent pebble sets for $Q_{i}$. Since this is true for all $1 \leq i \leq 3$, we have that $S \cap Q_{i}$ and $T \cap Q_{i}$ are equivalent pebble sets for all $i$. Thus $\pi$ is well defined.

To show that $\pi$ is one-to-one, suppose that $S, T \in \mathcal{S}(P, p)$ are two pebble sets such that $S \cap Q_{i}$ and $T \cap Q_{i}$ are equivalent pebble sets in all the $Q_{i}(1 \leq i \leq 3)$. Let $q \in S$ be any point. Since $S$ and $T$ both contain $p$, we may assume that $q \neq p$. Since the $Q_{i}$ cover $P$, there exists some $i$ such that $p \in Q_{i}$. Since $S \cap Q_{i}$ and $T \cap Q_{i}$ are equivalent pebble sets in $Q_{i}$, then there exists a point $r \in T \cap Q_{i}$ that lies in the same chamber of $Q_{i}$ as $q$. But by Lemma 20, the chamber of $Q_{i}$ that contains $q$ is also a chamber of $P$. Thus $q$ and $r$ lie in the same chamber of $P$. Since $q$ was chosen arbitrarily, we have that $S$ and $T$ are equivalent pebble sets in $P$.

To show that $\pi$ is onto, let $S_{1}, S_{2}$, and $S_{3}$ be, respectively, pebble sets in $Q_{1}$, $Q_{2}$, and $Q_{3}$ that contain $p$. We show that $S=S_{1} \cup S_{2} \cup S_{3}$ is a pebble set in $P$ by applying Lemma 3. Let $e$ be any edge and $v$ any vertex of $P$. We may assume that $v$ is an endpoint of an edge in $E_{1}$, for an identical argument applies if $v$ is an endpoint of an edge in $E_{2} \cup E_{3}$. If $e$ is an edge of $Q_{1}$, then since $S_{1}$ is a pebble set in $Q_{1}, \Delta e v$ contains precisely one point of $S_{1}$. Furthermore, $\Delta e v$ cannot contain a point of $S_{2}$. For the only way that $\Delta e v$ intersects $Q_{2}$ is if $e=e_{3}$, in which case the facts that $p \in \Delta e_{3} f_{2}$ and that $S_{2}$ is a pebble set in $Q_{2}$ reveal that $\Delta e v \cap Q_{2}$ contains no point of $S_{2}$. By similar reasoning, $\Delta e v$ contains no point of $S_{3}$.

Now if $e$ is not an edge of $Q_{1}$, then it is an edge in $E_{2} \cup E_{3} \cup\left\{e_{1}\right\}$. First consider that $e=e_{1}$. Then the interior of $\Delta e v \cap Q_{1}$ is a subset of $\Delta f_{1} v$. But $p \in \Delta f_{1} v$, and $S_{1}$ is a pebble set in $Q_{1}$. Thus $\Delta e v$ contains no point of $S_{1}-\{p\}$. Furthermore, since $p \in \Delta e_{1} f_{2}$ and $S_{2}$ is a pebble set in $Q_{2}$, then $\Delta e v$ contains no point of $S_{2}-\{p\}$. 
Similarly, since $p \in \Delta e_{1} f_{3}$ and $S_{3}$ is a pebble set in $Q_{3}$, then $\Delta e v$ contains no point of $S_{3}-\{p\}$. Thus $\Delta e v$ contains at most one point of $S$.

Finally, consider that $e \in E_{2} \cup E_{3}$. We may assume $e \in E_{2}$, for an identical argument will apply if $e \in E_{3}$. By Lemma 19, $\Delta e v$ contains no point of $S_{1}$. Since $\Delta e v$ does not intersect $Q_{3}$, then $\Delta e v$ contains no point of $S_{3}$. Finally, consider the quadrilateral determined by $f_{2}$ and $e$. This quadrilateral contains precisely two points of $S_{2}$, one of which is $p$. Since $e \notin \operatorname{ES}(C)$, we have that $p \notin \Delta e v$. Thus $\Delta e v$ contains at most one point of $S_{2}$.

We therefore have that every triangle of the form $\Delta e v$ contains at most one point of $S$, so that by Lemma 3,S is a pebble set in $P$. Thus $\pi$ is onto.

We are now ready to prove the converse of Theorem 17. If $C$ is a chamber with support size three, and we let $p$ be any point in $C$, then $p$ is close to an edge in each of the $Q_{i}$. Theorem 4 assures us that there are pebble sets in each of the $Q_{i}$ that contain $p$. By the proof of Theorem 21, the union of these pebble sets is a pebble set in the polygon. Thus we arrive at the following.

Corollary 22 A chamber with support size three is viable.

With the help of the next proposition, we can count the number of pebble sets that contain a point in a given chamber, where all other points lie in the periphery of the polygon.

Proposition 23 Let $C$ be a viable chamber in a polygon $P$, and let $S$ be a pebble set in $P$ that contains a point $p \in C$. Then the $S \cap Q_{i}$ are peripheral pebble sets in $Q_{i}$ if and only if all points of $S-\{p\}$ lie in the periphery of $P$.

Proof First suppose all the $S \cap Q_{i}$ are peripheral pebble sets in the $Q_{i}$. Now the only border triangles of $Q_{i}$ that are not also border triangles of $P$ must be defined by $f_{i}$ and an edge of $P$ incident with $f_{i}$. Such a triangle contains $p$. Thus if $q \in S-\{p\}, q$ lies in a border triangle of some $Q_{i}$ that is also a border triangle of $P$. Conversely, if every point $q \in S-\{p\}$ lies in the periphery of $P$, then for any $i$, either $q$ lies in the periphery of $Q_{i}$, or it lies outside $Q_{i}$.

By Proposition 23, the bijection in Theorem 21 can be restricted to a bijection between pebble sets in $P$ that contain a point in $C$ and where all other points lie in the periphery, and ordered triples of peripheral pebble sets in the $Q_{i}$ that contain a point close to the edge $f_{i}$. With Theorem 4 , we now have a way to construct all pebble sets in $P$ that have a point in $C$ and where all other points lie in the periphery. The following corollary allows us to determine the number of such pebble sets.

Corollary 24 Let $C$ be a chamber of a polygon $P$ such that $|\operatorname{ES}(C)|=3$. Define $E_{i}$ and $Q_{i}$ as in Construction 2, and let $n_{i}$ be the number of distinct endpoints of the edges of $E_{i}$. Let $D=\sum_{i=1}^{3} d\left(Q_{i}\right)$, where $d\left(Q_{i}\right)$ is defined as in Corollary 5. Then the number of pebble sets in $P$ containing $p$ and with all other points in the periphery is $2^{n-6+D}$. 
Proof Each $Q_{i}$ contains $n_{i}+2$ vertices, and $n_{1}+n_{2}+n_{3}=n$. By Theorem 21 and Corollary 5 , the number of such pebble sets is $\prod_{i=1}^{3} 2^{n_{i}-2+d\left(Q_{i}\right)}=2^{n-6+D}$.

\section{Finding Viable Chambers}

In this section we approach the task of finding the viable chambers of a polygon in two slightly different ways. First, given a particular chamber in a polygon, we show that determining whether its support size is three is surprisingly easy. Second, given an arbitrary $n$-gon, we describe an algorithm for finding all viable chambers that is $O\left(n^{3}\right)$. This represents an improvement over checking every chamber, for as we will show, the number of chambers in an $n$-gon is $O\left(n^{4}\right)$.

\subsection{A Second Characterization of Viability}

According to Proposition 18, only triangular chambers are viable. The converse, however, is not true in general. But if $C$ is a triangular chamber, and the chords and edges that determine the boundary of $C$ are incident with edges of the polygon in a particular way we will describe, then we can conclude that $|\operatorname{ES}(C)|=3$ and that therefore $C$ is viable.

One direction of claim 1 from the proof of Theorem 11 says that if either $e_{k} \in$ $\mathrm{ES}(C)$ or $e_{k-1} \in \operatorname{ES}(C)$, then $f \in \operatorname{ES}\left(C^{\prime}\right)$. Equivalently, if $f \notin \operatorname{ES}\left(C^{\prime}\right)$, then neither $e_{k}$ nor $e_{k-1}$ is in $\operatorname{ES}(C)$; and furthermore, by claim 2a in the proof of Proposition 11, every other edge of $P$ is in $\operatorname{ES}(C)$ if and only if it is in $\operatorname{ES}\left(C^{\prime}\right)$. The important point to be made here is that if $f \notin \operatorname{ES}\left(C^{\prime}\right)$, then $\operatorname{ES}\left(C^{\prime}\right)=\operatorname{ES}(C)$.

We may apply this result inductively on a set of consecutive edges in a polygon to have the following.

Lemma 25 Let $E=\left\{e_{i}, e_{i+1}, \ldots e_{j}\right\}$ be any set of consecutive edges of a polygon $P$, where $2 \leq|E| \leq n-2$. Let $g$ be the chord $v_{i} v_{j+1}$, and $C$ be any chamber of $P$ that does not lie in the polygon determined by $E$ and $g$. Let $P^{\prime}$ be the polygon determined by $g$ and the edges of $P$ not in $E$. Let $C^{\prime}$ be the chamber of $P^{\prime}$ that contains $C$. Finally, suppose that $g \notin \operatorname{ES}\left(C^{\prime}\right)$. Then $\operatorname{ES}\left(C^{\prime}\right)=\operatorname{ES}(C)$.

Now we demonstrate how to determine if a triangular chamber has support size three. Let $C$ be a triangular chamber in a polygon $P$, and suppose $f$ is an edge or chord that determines part of the boundary of $C$. In the proof of Proposition 18, we noted that the two edges of $P$ that are incident with $f$ and lie on the same side of $f$ as $C$ are edges in $\operatorname{ES}(C)$.

So consider all the edges of $P$ with the property that they are incident with an edge or chord that bounds $C$ and lie on the same side of the chord as $C$. Call this set of edges the boundary support of $C$, which we will denote $\operatorname{BS}(C)$. Then by the observation in the previous paragraph, we have that $\mathrm{BS}(C) \subseteq \operatorname{ES}(C)$.

Proposition 26 Suppose $C$ is a triangular chamber in an $n$-gon $P$, and suppose that $\mathrm{BS}(C)$ contains precisely three edges. Then $\mathrm{BS}(C)=\mathrm{ES}(C)$. 
Proof Let $\left\{f_{1}, f_{2}, f_{3}\right\}$ be the chords that determine the boundary of $C$, and write $\operatorname{BS}(C)=\left\{e_{1}, e_{2}, e_{3}\right\}$, where $f_{i}$ is incident with $e_{j}$ and $e_{k}(i, j, k$ distinct $)$. Let $V$ be the set of endpoints of the $f_{i}$. Note that $3 \leq|V| \leq 6$. First consider that $|V| \leq 5$, so that at least two of the $f_{i}$ are incident. Now the intersections of pairs of the $f_{i}$ determine the vertices of $C$. We thus have that a vertex of $P$ is also a vertex of $C$, so that $C$ lies in the periphery. In this case, $\operatorname{ES}(C)$ contains precisely three edges, so that $\mathrm{BS}(C)=\mathrm{ES}(C)$.

We may assume, therefore, that $|V|=6$. Let $P^{\prime}$ be the convex hull of $\operatorname{BS}(C)$. Since $|V|=6$, we have that $P^{\prime}$ is a hexagon, and $C$ is a chamber in the core of $P^{\prime}$. (See Fig. 7.) Each $f_{i}$ is a chord of $P$, and the perimeter of $P^{\prime}$ consists of $\left\{e_{1}, e_{2}, e_{3}, g_{1}, g_{2}, g_{3}\right\}$, where the $g_{i}$ are chords of $P$ joining $e_{j}$ and $e_{k}(i, j$, and $k$ all distinct). The perimeter of $P^{\prime}$ naturally partitions the edges of $P$ into six subsets: $\left\{e_{1}\right\},\left\{e_{2}\right\},\left\{e_{3}\right\}, E_{1}, E_{2}$, and $E_{3}$, as illustrated in Fig. 7. Note that the $E_{i}$ are all nonempty. We show that no edge of the $E_{i}$ is in the edge support of $C$.

Let $1 \leq i \leq 3$, and let $P_{i}$ be the polygon formed from $P$ by removing the edges of $E_{i}$ and including $g_{i}$. Now $C$ is a chamber in both $P$ and $P_{i}$. We claim $g_{i} \notin \operatorname{ES}(C)$ in $P_{i}$. If $j \neq i$, then $C$ lies in the quadrilateral defined by $e_{i}$ and $e_{j}$. Thus $C$ lies in no triangle determined by $g_{i}$ and an endpoint of any edge in $E_{j}$, so that $g_{i} \notin \operatorname{ES}(C)$ (in $P_{i}$ ). Thus by Lemma 25, no edge in $E_{i}$ is in $\operatorname{ES}(C)$ (in $P$ ). Since this is true for $1 \leq i \leq 3$, we have that $\operatorname{ES}(C)=\left\{e_{1}, e_{2}, e_{3}\right\}$.

If a chamber is not triangular, its boundary support must contain more than three edges. With this and Propositions 18 and 26, we have the following.

Theorem 27 A chamber is viable if and only if its boundary support contains precisely three edges.

\subsection{An Algorithm for Determining all Viable Chambers}

To determine all viable chambers of a given $n$-gon, one approach would be to check every chamber to see if satisfies the criterion of Theorem 27. For an $n$-gon in general position (so that no three chords intersect at a single point), the number of chambers is $\left(\begin{array}{c}n-1 \\ 2\end{array}\right)+\left(\begin{array}{c}n \\ 4\end{array}\right) .{ }^{1}$ Therefore, any algorithm that checks every chamber for viability will generally run in $O\left(n^{4}\right)$ time.

This can be improved in the following way. Select three edges of the $n$-gon, and denote them $E=\left\{e_{i}, e_{j}, e_{k}\right\}$. In this subsection, we will describe a decision procedure for determining when $E$ is the edge support for some chamber of $P$.

Let $P^{\prime}$ be the convex hull of $E$. If $P^{\prime}$ is a quadrilateral or pentagon, then $E$ is the edge support of a peripheral chamber of $P$, which is viable. If $P^{\prime}$ is a hexagon such

\footnotetext{
${ }^{1}$ The number of chambers in $P$ can be determined by considering the graph $G$ whose vertices are the intersections of all the chords and edges of $P$, and whose edges are the edges of $P$ and segments of the chords joining two points of intersection. We note that there is precisely one intersection of chords for every choice of four vertices of $P$, so that the number of vertices of $G$ is $n+\left(\begin{array}{l}n \\ 4\end{array}\right)$. Every vertex of $P$ has degree $n-1$ and the remaining vertices of $G$ have degree four. Since the sum of the degrees of the vertices of $G$ is twice the number of edges, we have that $G$ contains $\left(\begin{array}{c}n \\ 2\end{array}\right)+2\left(\begin{array}{l}n \\ 4\end{array}\right)$ edges. Applying Euler's formula to this planar graph, the number of chambers in $P$ is therefore $\left(\begin{array}{c}n-1 \\ 2\end{array}\right)+\left(\begin{array}{c}n \\ 4\end{array}\right)$.
} 
that the chords that connect opposite vertices all meet at one point, then no chamber of $P^{\prime}$ (and therefore no chamber of $P$ ) has $E$ as its edge support (as can be seen by considering boundary support instead).

If $P^{\prime}$ is a hexagon in general position, then $P^{\prime}$ contains a triangular chamber $C$ in the core. If $\mathrm{BS}(C)=E$, then $C$ is a viable chamber of $P^{\prime}$, as illustrated in Fig. 4. Furthermore, $C$ is also a viable chamber in $P$, as the following theorem demonstrates. For notational simplicity, we let $\left\{e_{1}, e_{2}, e_{3}\right\}$ represent any choice of three edges of the $n$-gon.

Theorem 28 Let $E=\left\{e_{1}, e_{2}, e_{3}\right\}$ be any three edges of an $n$-gon $P$, and let $P^{\prime}$ be the convex hull of E. Suppose $P^{\prime}$ is a hexagon in general position, so that it contains a triangular chamber $C$ in the core. Finally, suppose that $\mathrm{BS}(C)=E$. Then $C$ is a viable chamber of $P$.

Proof Let $\left\{g_{1}, g_{2}, g_{3}\right\}$ be the other three edges of $P^{\prime}$, and let $E_{1}, E_{2}$, and $E_{3}$ be the edge subsets of $P$ as labeled in Fig. 7. First we show that $C$ is a chamber of $P$ by supposing that $D$ is a chamber of $P$ that is a proper subset of $C$. Then there is a chord $f$ of $P$ that is not a chord of $P^{\prime}$ that intersects $C$. Clearly at least one endpoint of $f$ is not a vertex of $P^{\prime}$. Thus this endpoint would determine an edge of $P$ in one of the $E_{i}$ that is in $\operatorname{BS}(D)$, hence in $\operatorname{ES}(D)$ in $P$. But then by Lemma $25, g_{i} \in \operatorname{ES}(C)$ in $P^{\prime}$, which is false. Thus $C$ is a chamber of $P$.

Let $P_{1}$ be the convex hull of $E \cup E_{1}$. By Lemma 25, since $g_{1} \notin \mathrm{ES}(C)$ in $P^{\prime}$, then $\operatorname{ES}(C)$ in $P_{1}$ is precisely the same as $\operatorname{ES}(C)$ in $P^{\prime}$. Now let $P_{2}$ be the convex hull of $E \cup E_{1} \cup E_{2}$. Again by Lemma 25, since $g_{2} \notin \operatorname{ES}(C)$ in $P_{1}$, then $\operatorname{ES}(C)$ in $P_{2}$ is precisely the same as $\operatorname{ES}(C)$ in $P_{1}$. Finally, since $g_{3} \notin \operatorname{ES}(C)$ in $P_{2}$, applying Lemma 25 a third time reveals that $\operatorname{ES}(C)$ in $P$ is precisely the same as $\operatorname{ES}(C)$ in $P_{2}$. Thus in $P, \operatorname{ES}(C)=E$ and by Corollary 22, $C$ is a viable chamber of $P$.

Since the number of such edge triples is $\left(\begin{array}{l}n \\ 3\end{array}\right)$, any algorithm that enumerates all viable chambers of $P$ using Theorem 28 will run in $O\left(n^{3}\right)$ time.

\section{Further Questions}

In this paper, we have made some progress in characterizing all pebble sets in a given polygon. First, we have a lower bound on the number of pebble sets, given by the $n 2^{n-5}$ peripheral pebble sets. This lower bound is actually achieved when there are no chambers outside the periphery whose support size is three-for instance, when the polygon is regular.

Stronger still, a point can be in a pebble set if and only if the support size of its chamber is three. And given such a chamber, we can use the construction in Sect. 4.3 to decompose the polygon into three subpolygons $Q_{1}, Q_{2}$ and $Q_{3}$. Theorem 21 assures us that finding pebble sets in each of the $Q_{i}$ is equivalent to finding a pebble set in the original polygon. This suggests an inductive approach to characterizing pebble sets. This inductive approach is algorithmic, but leaves much to be desired. It seems inefficient, but worse, it does not seem to capture the entire structure of the problem. 
The set of chambers whose support sizes are all three does seem to relate to the corresponding sets for the $Q_{i}$, and it might be possible to use this fact to extract a more refined object that makes counting pebble sets easier.

For instance, we showed that if $p$ lies in a viable chamber in the core, then we can easily count the number of pebble sets whose only core point is $p$. What is the corresponding description for two core points? Or more generally?

Beyond this main question, we could seek solutions to related problems. First, the statement of the problem by Lukácz and András specified that there should be $n-2$ points in the required set and that a point on the boundary of a triangle does not count as being in that triangle. We showed in Proposition 1 that as a result, points in a pebble set may not lie on chords of the polygon. But if we allow more points, or if we change how we count points on the boundary of a triangle, this proposition no longer holds. For instance, if the polygon is a square, we can augment any pebble set by adding a point in the center of the square, which does not count toward any total since it does not lie in any triangle. On the other hand, if we decide that points on the boundary of the triangle count as lying in the triangle (so that the triangles are closed), we could have a set with one point (the center of the square) with the required property. If we count points on the boundary of a triangle as providing $1 / 2$ a point to that triangle, we could have sets with two points, both straddling the same chord but from opposite sides of the center. The structure of these sets seems less clear.

Another obvious generalization would be to consider polytopes in higher dimensions, as in [3], replacing the notion of triangle with that of simplices. But there are many cases where the problem analogous to the polygon question has no solution. For instance, in three dimensions, consider the triangular bipyramid-that is, the union of two tetrahedra that share a face. This has a triangulation into two simplices (the two tetrahedra used to define it), and another triangulation into three simplices, where the simplices all surround the edge between the apexes of the tetrahedra. As a result, if each tetrahedron contains a point, then we are left with requiring a set with two points and three points simultaneously, which is impossible. Thus, the structure of this problem seems very different.

One way around this is to insist that every simplex has at most one point, which is precisely the definition of pebble set in [3]. It is likely that any generalization to higher dimensions should use this notion.

Another generalization in the plane would be to insist that every triangle contain $k$ points, for a given integer $k \geq 1$. Some preliminary investigations have indicated that this problem has some interesting structure, though this problem seems to have a character very different from the problem posed in this paper.

\section{References}

1. András, A., Lukácz, S.: Problem 10936. Am. Math. Mon. 109, 392 (2002)

2. Cahill, N.: Convex $n$-gons. Am. Math. Mon. 110, 545f (2003)

3. De Loera, J.A., Peterson, E., Su, F.: A polytopal generalization of Sperner's lemma. J. Comb. Theory Ser. A 100, 1-26 (2002) 